Fecha de recepción: julio 2011 Fecha de aceptación: octubre 2011 Versión final: abril 2012

\section{La Pequeña y Mediana empresa y la función de la comunicación}

Patricia Iurcovich *

\begin{abstract}
Resumen: El artículo aborda en su desarrollo la problemática de las pequeñas y medianas empresas a la hora de incorporar en sus estructuras estrategias de comunicación. Que las comunicaciones integradas, campañas de publicidad e imagen, Responsabilidad Social Empresaria y otros componentes de la comunicación pertenecen sólo a las grandes Corporaciones es un mito que aún subsiste en el imaginario empresarial. En un mercado competitivo y complejo, todos tienen una estrategia, implícita o explícita, buena o mala, a corto o largo plazo y cada organización tiene que competir contra esas otras estrategias. La ventaja competitiva surge de tener un plan y una metodología superior a la de la competencia y un equipo humano altamente capacitado. Las comunicaciones integradas de marketing bien gestionadas pueden realizarse a un costo accesible a cualquier empresa por pequeña que sea y con un alto rendimiento siempre y cuando estén gestionadas por profesionales que puedan diseñar un plan eficaz con las herramientas adecuadas para cumplir los objetivos esperados..
\end{abstract}

Palabras clave: comunicaciones integradas - gestión comunicacional - imagen corporativa Pyme - redes sociales.

[Resúmenes en inglés y portugués en la página 84]

${ }^{(*)}$ Master en Comunicación Institucional (UCES). Licenciada en Publicidad y Analista en Medios (Universidad del Salvador). Profesora de la Universidad de Palermo en el Departamento de Comunicación Comunicación Corporativa - Empresa de la Facultad de Diseño y Comunicación. Es docente en otras Universidades e Instituciones. [Ver CV completo en página 140]

Existe un mito por el cual las comunicaciones integradas, campañas de publicidad e imagen, Responsabilidad social empresaria y otros componentes de la comunicación pertenecen sólo a las grandes Corporaciones. Esto no es así. En todo caso habrá que ver cuál es el uso que se le da a dichas herramientas y en qué lugar se ubica la imagen institucional en la tabla de orden de las Pymes.

En un mercado competitivo, complejo, con crisis como las que venimos observando y siendo protagonistas en ésta última década, todos tienen una estrategia, implícita o explícita, buena o mala, a corto o largo plazo, con segmentación de mercado o no, etc. y cada Organización tiene que competir contra esas otras estrategias en el contexto descrito. La ventaja competitiva 
surge de tener un plan y una metodología superior a la de la competencia y un equipo humano altamente capacitado.

Es una forma de afirmar lo obvio: quien no se contacta con el medio exterior tiende a su desaparición.

En la actualidad muchas PyMEs han podido encontrar en estos nuevos escenarios una salida: reconvertirse según el contexto y ofertar según la nueva demanda. Lo pudieron hacer porque llevaron a cabo las gestiones necesarias utilizando su capacidad de acción.

Las empresas que han detectado oportunidades y que modernizado su línea de producción y sus cuadros gerenciales entraron al nuevo escenario con fuerza y protagonismo. Poder sostener la marcha, crecer y afianzarse, es un trabajo de largo plazo que merece ciertas consideraciones. Pero también estas empresas sirven como modelos a copiar por otras PyMEs.

Cuando se repasa la bibliografía de diagnóstico y gestión de las PyMEs, nos encontramos con que antes de la década del '90, estas empresas eran sinónimo de atraso, económicamente pequeñas para constituir una fuerza en el PBI y por supuesto con un sistema de producción que no constituía ninguna pauta de calidad.

Hoy, luego de la década de la convertibilidad, las PyMEs se comienzan a levantar como posibles promotoras de la producción industrial y de servicios.

La gran empresa ha entrado en crisis porque ha entrado en crisis todo lo que expresa uniformidad y ritmos autoritariamente establecidos, jerarquías rígidas y encuadres rudamente normativos. Cuando el desafío se expresa en necesidades de flexibilidad, descentralización, desburocratización, liderazgos horizontales, espacios compartidos, agilidad y adaptaciones rápidas, personalización de la gestión y del consumo, artesanía y trabajos a medida o de series reducidas; las PYMES emergen con una identidad clara, natural, más consonante a todos estos conceptos que la gran empresa, quien estimula, desde su gigantismo, una reconversión exógena para las Pequeñas y medianas empresas.

El desafío de las pequeñas y medianas empresas esta en la recuperación de una identidad, en la revalorización de su cultura organizacional como base de la innovación. Estas empresas tienen ventajas comparativas desde lo cultural: confianza en el éxito, personalismos inquebrantables ante los vaivenes económicos, métodos artesanales de gestión, etc.

\section{¿En qué contribuyen las Comunicaciones Integradas?}

Las Pymes tienen muchos problemas para administrar los escasos recursos de comunicación que tienen para sus empresas ya sea porque gastan en campañas que no son las adecuadas ó porque van dirigidas no siempre al publico apropiado desperdiciando tiempo y dinero; o bien porque no invierten en ninguna campaña de comunicación. Las comunicaciones integradas de marketing bien gestionadas pueden realizarse a un costo accesible a cualquier empresa por pequeña que sea y con un alto rendimiento siempre y cuando estén gestionadas por profesionales que puedan diseñar un plan eficaz con las herramientas adecuadas para cumplir los objetivos esperados. En el concepto de identidad que ésta ligado a la misión y visión de la empresa, en la relación con el cliente, en el packaging del producto, en la relación con los empleados, por ejemplo. Todas éstas son acciones y funciones que las herramientas del marketing, la publicidad, la comunicación organizacional, pueden potenciar, haciendo más eficaces y eficientes los resultados. 
Es relativamente cierto que éstas herramientas eran consideradas sólo para las grandes empresas, ¿̇las razones?, muchas. Pero entre ellas el alto costo y la falta de profesionalización de las PyMEs. La adecuación gerencial de estas empresas a los nuevos escenarios, puede constituir un proceso que enriquezca el desarrollo más general de la sociedad, anclado en la revalorización de lo cultural, de la diversidad, y de una nueva trama de relaciones socio - comunitarias orientadas a una mejor calidad de vida.

Las ventajas comparativas con que cuentan como: -una eficaz relación empresa - producto cliente; una fidelización alta del cliente y una flexibilidad ante las demandas del público hacia un producto; entre otras, hacen de la PyME un importante nexo entre organización/comunidad.

Tal es así que muchos teóricos del management comienzan a pensar que las grandes empresas deben constituirse a partir de pequeñas empresas células.

Si las pequeñas y medianas empresas pudieran crear valor agregado a sus organizaciones y a sus productos: como el valor marca, el packaging con un diseño estético y/o reforzar sus vínculos con los clientes internos y externos mediante el CRM, etc. Podrían establecer un valor de gestión de una gran empresa, añadiendo una capacidad de flexibilidad y relaciones con la comunidad. Es bien sabido que no es sencillo gestionar la comunicación en estas empresas. En principio porque la venta de modelos de gestión apelando al éxito logrado en contextos organizacionales de gran alcance, no contiene garantías de éxito. Por otro lado, los altos costos que ello implica, la poca inversión, la reticencia a iniciar planes de reingeniería por parte del Estado para éstas, el contexto de incertidumbre económica y las particularidades organizacionales/culturales con que cuentan, no resulta del todo viable la incorporación de las comunicaciones integradas.

Para dar un paso hay que tener decisión, para dar 100 pasos hay que dar primero uno. No se trata de encontrar las diferenciaciones especulativas, sino de encontrar las articulaciones que eludan las superposiciones de roles, tareas y funciones y transformemos nuestros conocimientos en acciones concretas y funcionales a un servicio, que en las PyMEs será la mejora continua del negocio.

\section{El aporte de las acciones sociales en las Pyme}

La década del '90 se caracterizó por la contribución que iniciaron las acciones sociales y de Responsabilidad social empresaria en las grandes Corporaciones y en las Pymes.

Planes de educación vial llevados a las escuelas del país, programas de protección y cuidado del medio ambiente, programas de aprendizaje en el ámbito de la alimentación son algunos de los publicados en los periódicos en lo que va del año.

La pregunta entonces que se haría Bernardo Kliksberg, economista argentino, pensador, asesor en temas de RSE en Naciones Unidas, argentino, es entonces, ¿Existe la ciudadanía corporativa?, ¿Existe la forma en que algún día las empresas dejen de convertirse en meros actores económicos para mejorar su perfil y actuar como verdaderos ciudadanos?.Las empresas corporativas acá y en el mundo hace tiempo que vienen trabajando en esta línea, desarrollando planes del cuidado del medio ambiente, contribuyendo con acciones filantrópicas en la comunidad, etc. Y porqué no, utilizar esta nueva modalidad y aplicarla a las PYMES. En la década del 2000, plena crisis en Argentina, el diseñador Martin Churba generó un proyecto por el cual financió a costureras del interior del país un proyecto para que comiencen a realizar uniformes y tejidos 
los que luego fueron exportados al mundo. Esto significa que si aún en términos de ventas, las empresas pueden satisfacer sus necesidades y además contribuir a tratar mejor a sus empleados, generar cooperativas, proyectos y convertirse en actores sociales, mucho mejor.

En los países desarrollados el Estado es el encargado de estas cuestiones y las empresas en menor medida, en los países emergentes, todo lo contrario. Las Pymes que logren incorporar estas cuestiones a su quehacer cotidiano además de satisfacer las necesidades lograrán mejorar su imagen institucional.

\section{La importancia de la comunicación interna en las Pymes}

En realidad primero deberíamos comenzar por hablar de la importancia de la capacitación a los empleados. No cabe posibilidad de pensar en comunicación si antes no podemos pensar en capacitación. Las personas que ingresan a trabajar en las Pymes no siempre atraviesan por selecciones de consultoras de recursos humanos. Se da mas la cultura del poder, y de la persona en donde prevalece más la confianza que el perfil de la misma, que a comparativa de las empresas grandes en donde se prioriza la cultura de la función y del poder, en donde pospuestos y posiciones jerárquicas ocupan otro espacio.

Algunas Pymes tienen diferentes niveles de informalidad que, en ocasiones, generan trastornos que pueden evitarse. Uno de ellos es la improvisación en materia de recibir o dar información a sus empleados. La comunicación ha crecido notablemente en estos últimos años, y básicamente en el sector Pymes y de emprendedores. Argentina, como la mayoría de los países europeos, por su tipo de economía, está haciendo cada vez más foco en facilitar los emprendimientos y retomar el desarrollo productivo de las Pymes como en años anteriores a la década del '90. Este fenómeno va lógicamente acompañado de la comunicación; sin ella sería muy difícil pensar en el negocio a solas. Cuando hablamos de comunicación no podemos no referirnos primero a la comunicación interna, cuyo sustento fundamental es la motivación y la información. Ambas deben ir juntas; hablar de comunicación interna es hablar de personas, de redes y sistemas de información sin los cuales el crecimiento del negocio seria impensable. No está escrito que todas las empresas deben estar inmersas en una plataforma de intranet, pero si está casi demostrado que cuando la comunicación interna falla, mucho más cuando se trata de las PYMES familiares como lo son la mayoría, todas las campañas de publicidad o de comunicación en las que podamos pensar son innecesarias. ¿Cómo se comunica un papá con su hijo en una Pyme? En que rol se pone cada uno al momento de establecer un vínculo? ¿Cómo se muestran hacia el afuera frente a un cliente? Se envían mails o simplemente almuerzan y se ponen de acuerdo en alguna estrategia?

Estas preguntas tan simple contribuyen a entender que para establecer mecanismos de información y transformar los canales informales de comunicación (boca a boca, almuerzos, etc), de las Pymes en canales formales(mails, carteleras) los actores intervinientes de las mismas deben sentir la necesidad de experimentar caudales de información en muchos casos similares a empresas grandes. Si una empresa como Cheeky creció tanto en estos últimos años, exportando, abriendo locales, dando charlas en Universidades, como tantas otras, Freddo, Café Martínez, etc, es porque decidió, imagino, atravesar por procesos de cambio en donde la comunicación entra a jugar un rol fundamental, sea interna como externa. 


\section{El aporte de las redes sociales}

Las redes sociales constituyen hoy por hoy la forma más directa y moderna a través de la cual se comunican las personas, medios, empresas, instituciones. Hoy se habla más de Twitter y Facebook que de datos como la dirección, mail o telefóno hasta de un comercio, una empresa. Las Pymes deben aggiornarse, muchas ya están en camino, otras aún, no; ó por falta de presupuesto ó porque aún no se sienten perteneciendo al mundo de la comunicación. Y sin darse cuenta tiene a mano muchas herramientas. La posibilidad de insertarse en una feria o exposición, la posibilidad de enviar un caso o establecer un lazo con una universidad y dar una charla, todas estas son herramientas de comunicación.

\section{Porqué el empresario Pyme entiende la comunicación como costo y no como inversión?}

Por último, ¿porqué para algunas PYMES la comunicación aún no es vista como una inversión y si como un costo? Porque para muchos empresario no se puede tangibilizar la comunicación y medirla es costoso, se llega hasta la creación de un logo, el inicio del posicionamiento de la marca, pero cuando se trata de meterse en redes sociales o iniciar una campaña de fidelización con los clientes o establecer mecanismos de información, todo se vuelve más volátil, tal vez, sea hora de empezar.

A continuación algunas reflexiones...

Todos hablan de la satisfacción al cliente; hasta los que no la logran, ni hacen, ni toman medidas adecuada para ello. La satisfacción al cliente es la única posibilidad de una empresa para poder seguir siendo tal. No obstante, ya no es suficiente. Hay que capacitarse y no tener miedo al cambio. La Pyme necesita de motor, de capital humano comprometido; en la empresa grande estos mecanismos están más estandarizados y se pueden llevar a cabo más fácilmente, porque hay presupuesto y porque el sistema lleva a eso, en la Pyme hay que construirlo día a día. Despertar al emprendedor que llevamos dentro En medio de un horizonte sombrío en cuanto al empleo y la situación económica de muchos países, la idea de ser dueños de nuestro propio destino es muy tentadora. ¿Pero cómo podemos lograrlo? No todos llevamos un emprendedor dentro que nos empuje a materializar el negocio con el que siempre habíamos soñado.

\section{Referencias Bibliográficas}

Schultz, D. y Tannenbaum, S. (1992). Comunicaciones Integradas. Mg Graw Hill. Cap II y III. Biblioteca Práctica de Administración de la pequeña y mediana empresa. Ed Océano Centrum. Cap II.

Elias, J. y Mascaray, J. Mas allá de la comunicación interna.

Trueba, I.; Carzola, A. y de Gracia, J. J. (1995). Proyectos Empresariales. Formulación, evaluación. Madrid: Ediciones Mundi-Prensa. 
Summary: The article approaches the problematic of small and medium companies at the time of incorporating communication strategies in its organizational structures. The commom knowledge that sustains that integrated communications, advertising and corporate image campaigns, Social corporate Responsibility and other components of the communication belong only to great Corporations is a myth that still survives in the corporate management imaginary. In a competitive and complex market, every organization has a strategy, implicit or explicit, good or bad, to short or long term and each organization must compete against others. Competitive advantage arises from having a plan and a methodology that surpasses the one of the competition and a human equipment highly enabled. Good managed integrated communications can be affordable to any company and with a high performance as long as they are managed by professionals who can design an effective plan with the adapted tools to fulfill the objectives awaited.

Key words: communicational management - corporate image - integrated communications small and medium companies - social networks.

Resumo: $\mathrm{O}$ artigo aborda a problemática das pequenas e medias empresas à hora de incorporar em suas estruturas estratégias de comunicação. Que as comunicações integradas, campanhas de publicidade e imagem, Responsabilidade Social Empressária e outros componentes da comunicação pertencem só às grandes corporações é um mito que ainda permanece no imaginário empressarial. Num mercado competitivo e complexo, todos têm uma estratégia, implícita ou explícita, boa ou má, a curto ou longo prazo e cada organização tem que competir contra essas outras estratégias. A vantagem competitiva surge de ter um plano e uma metodologia superior à da comepetência e um equipe humano altamente capacitado. As comunicações integradas de marketing bem gestionadas podem realizar-se com um custo accesível a cualquer empressa por pequena que seja e com um alto rendimento sempre e quando estejam gestionadas por profissionais que possam desehnar um plano eficaz com as ferramentas adequadas para cumprir os objetivos esperados.

Palavras chave: comunicações integradas - gestão comunicacional - imagem corporativa Pyme - redes sociais. 